\title{
On the jets, kinks, and spheromaks formed by a planar magnetized coaxial gun
}

\author{
S. C. Hsu a) and P. M. Bellan ${ }^{\text {b) }}$ \\ California Institute of Technology, Pasadena, California 91125
}

(Received 26 May 2004; accepted 4 November 2004; published online 4 February 2005)

\begin{abstract}
Measurements of the various plasma configurations produced by a planar magnetized coaxial gun provide insight into the magnetic topology evolution resulting from magnetic helicity injection. Important features of the experiments are a very simple coaxial gun design so that all observed geometrical complexity is due to the intrinsic physical dynamics rather than the source shape and use of a fast multiple-frame digital camera which provides direct imaging of topologically complex shapes and dynamics. Three key experimental findings were obtained: (1) formation of an axial collimated jet [Hsu and Bellan, Mon. Not. R. Astron. Soc. 334, 257 (2002)] that is consistent with a magnetohydrodynamic description of astrophysical jets, (2) identification of the kink instability when this jet satisfies the Kruskal-Shafranov limit, and (3) the nonlinear properties of the kink instability providing a conversion of toroidal to poloidal flux as required for spheromak formation by a coaxial magnetized source [Hsu and Bellan, Phys. Rev. Lett. 90, 215002 (2003)]. An interpretation is proposed for how the $n=1$ central column instability provides flux amplification during spheromak formation and sustainment, and it is shown that jet collimation can occur within one rotation of the background poloidal field. (C) 2005 American Institute of Physics.
\end{abstract}

[DOI: $10.1063 / 1.1850921]$

\section{INTRODUCTION}

There has long been a mutually beneficial exchange of ideas between laboratory plasma physics and plasma astrophysics. An example is the proposal by Lundquist ${ }^{1}$ that cosmical magnetic fields are force-free, ${ }^{2}$ satisfying $\nabla \times \mathbf{B}=\lambda \mathbf{B}$. Woltjer showed ${ }^{3}$ that these force-free states are intimately related to the concept of magnetic helicity conservation and that the force-free state results from a variational principle in which the magnetic energy of a system is minimized subject to the constraint of constant magnetic helicity, which is a measure of magnetic flux twist and linkage. ${ }^{4}$ This idea was then used to explain the spontaneous generation of reversed magnetic fields in the reversed field pinch ${ }^{5}$ and the tendency of coaxial gun produced plasmas to reach the spheromak state. ${ }^{6}$ Although Taylor relaxation, ${ }^{7}$ as this process is now known, is not a complete and rigorous theory, it was very successful, indicating that the same fundamental physical principles govern both laboratory and astrophysical plasmas. The Taylor argument showed that the end result of complex dynamics is a relatively simple relaxed state, but the argument sidesteps the issue of characterizing the detailed dynamical processes leading to this state. This paper describes recent experiments providing insights into the detailed dynamical processes of magnetic helicity injection and plasma relaxation. In particular, the experiments shed light on the nonaxisymmetric dynamics of spheromak formation, a subject with a long history beginning with the work of Alfvén ${ }^{8}$ and Lindberg. ${ }^{9}$ These experiments also show how magnetic

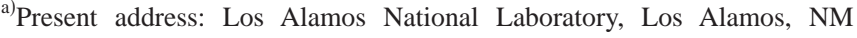
87545. Electronic mail: scotthsu@lanl.gov

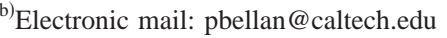

helicity injection can lead to the formation and collimation of magnetically driven jets in astrophysics. ${ }^{10-12}$

An important feature of this experiment is the use of the simplest possible setup for allowing studies of the details and three-dimensional (3D) dynamics of magnetic helicity injection and spheromak formation. Key results include (1) observation of distinct plasma regimes depending on the parameter $\lambda_{\text {gun }}$ (ratio of peak gun current to flux), similar to those previously found ${ }^{13}$ using a conventional cylindrical coaxial gun, (2) formation and axial expansion of a central plasma column from merging of plasma-filled poloidal flux tubes ${ }^{14}$ in a manner consistent with magnetohydrodynamic (MHD) models of magnetically driven astrophysical jets, ${ }^{12,15-17}$ (3) clear identification of a kink instability of this central column and a kink onset threshold that is quantitatively consistent with the Kruskal-Shafranov limit for an ideal MHD kink, ${ }^{14,18}$ and (4) identification of the nonlinear limit of the kink instability as a poloidal flux amplification mechanism leading to spheromak formation. ${ }^{18}$

This paper is organized as follows. Section II describes the experimental setup. Section III presents the experimental results. Section IV provides a detailed discussion of the results in the context of both spheromak formation and astrophysical jets. A summary is given in Sec. V.

\section{EXPERIMENTAL SETUP}

In order to facilitate interpretation of results, the coaxial gun design was made to be as geometrically simple as possible: a disk surrounded by a coplanar annulus [Fig. 1(a)] and a bias magnetic field coil located just behind the disk [not visible in Fig. 1(a)]. This experimental setup provided the additional advantage that the entire plasma formation region is in plain view so that all steps of the topological evolution 
(a)

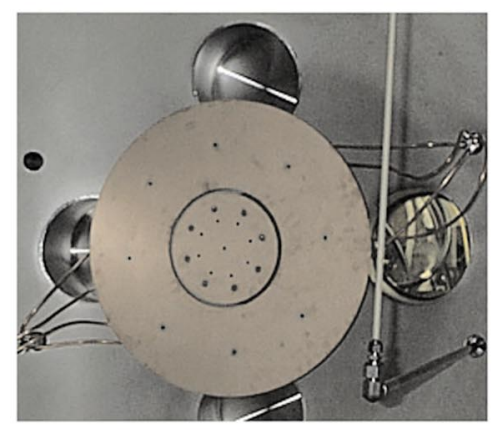

(b)

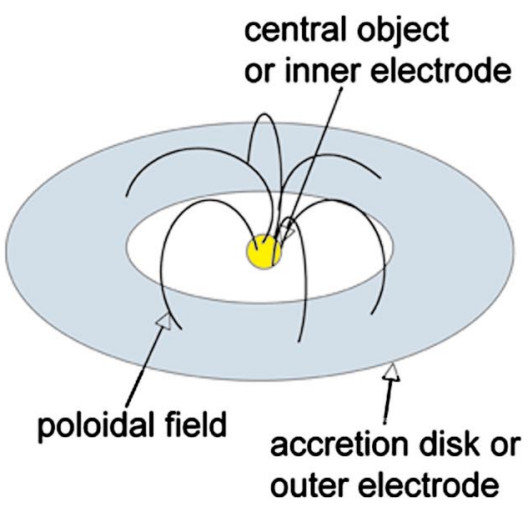

(c)

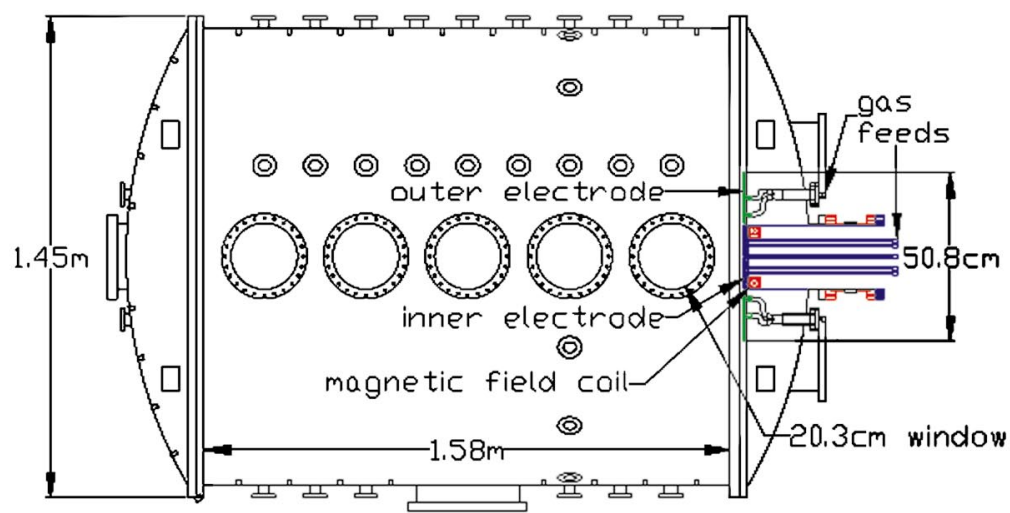

(d)

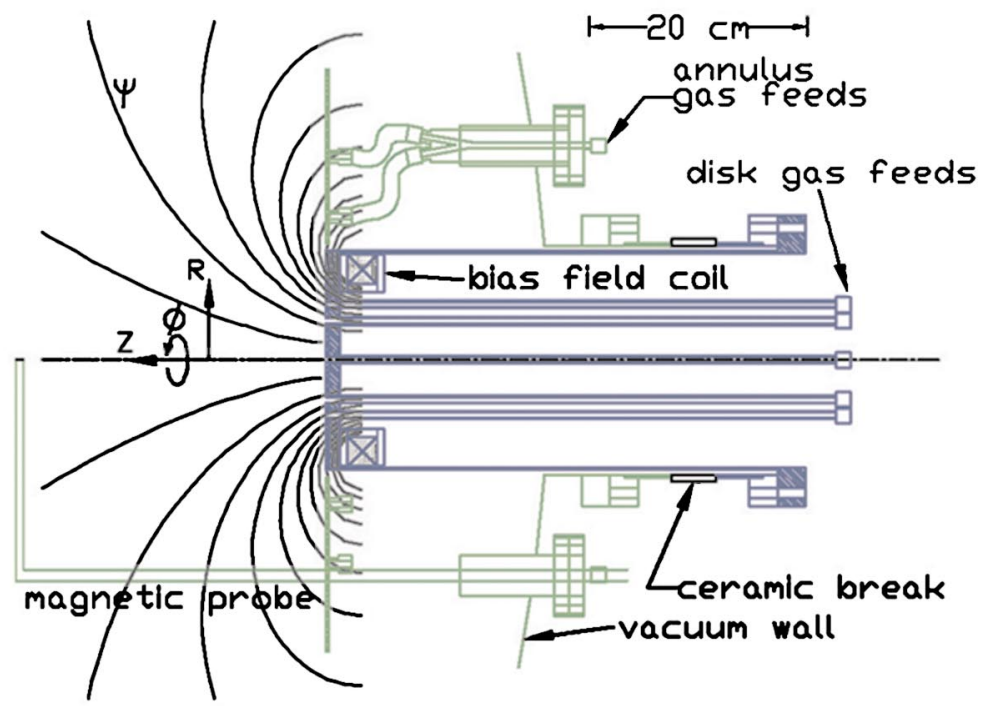

FIG. 1. (Color). (a) End-view photograph of coaxial gun and rotatable magnetic probe array. (b) Schematic of essential ingredients of both a coaxial gun and astrophysical star-disk system. (c) Large vacuum chamber with multiple diagnostic ports and coaxial gun mounted on right end dome. (d) Side-view schematic of planar coaxial gun, showing inner electrode (blue), outer electrode (green), gas feed lines, contours of constant bias poloidal flux, and cylindrical coordinate system.

could be observed. Note that this setup has essentially the same magnetic topology as a star-disk system associated with magnetically driven astrophysical jets [see Fig. 1(b)]. The various components of the experimental setup are described in detail below.

\section{A. Vacuum chamber}

The cylindrical vacuum chamber, shown in Fig. 1(c), is about $2 \mathrm{~m}$ long, $1.4 \mathrm{~m}$ in diameter, and constructed using $6 \mathrm{~mm}$ thick electropolished stainless steel. The chamber has large windows on the side that provide a good view of the entire plasma. A key point is that the chamber size is large compared to both the coaxial gun source and the plasma, so that plasma-wall interactions are minimal during the time of interest for the results presented here. The chamber is evacuated to $1.5 \times 10^{-7} \mathrm{~T}$ with a cryogenic pump, and a sorption pump is used for initial pump-down from atmosphere to $100 \mathrm{mT}$ so that the the entire pumping system is oil-free. The oil-free property and the minimal plasma-wall interactions resulted in plasmas that did not require a discharge cleaning protocol in order to provide clean, reproducible results.

\section{B. Planar magnetized coaxial gun}

The planar magnetized coaxial gun, ${ }^{19}$ shown in Figs. 1(a) and 1(d), is mounted on one end dome of the vacuum chamber, as shown in Fig. 1(c). The gun consists of (1) an inner $20.3 \mathrm{~cm}$ diameter copper disk electrode, (2) a coplanar, coaxial outer $50.8 \mathrm{~cm}$ diameter copper annulus electrode, and (3) a magnetic field coil located just behind the inner electrode. The copper disk inner electrode is bolted onto the vacuum face of a reentrant $7.5 \mathrm{in}$. diameter stainless steel half nipple mounted on one end of an 8 in. diameter ceramic break which insulates the inner electrode from ground. The other end of the ceramic break is mounted on a 10 in. flange centered on the vacuum chamber end-dome. Thus, the inner electrode is electrically floating and is connected to a capacitor bank via coaxial cables connected across the ceramic insulating break. The outer annulus electrode is mounted via 


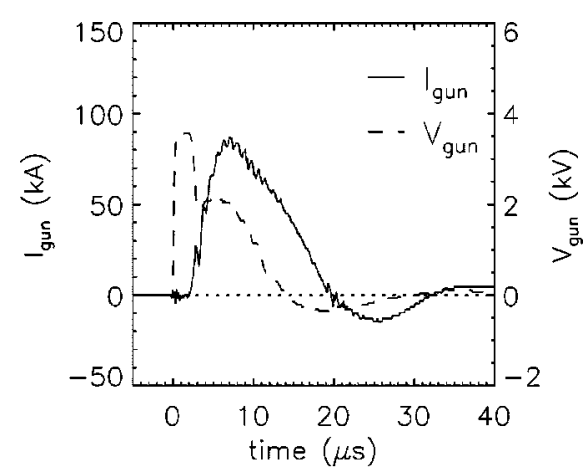

FIG. 2. Typical $I_{\text {gun }}$ (solid) and $V_{\text {gun }}$ (dashed) traces (shot 1210). Breakdown occurs at $t=2-3 \mu \mathrm{s}$ when $V_{\text {gun }}$ drops and $I_{\text {gun }}$ rises.

copper brackets directly to the inner surface of the vacuum chamber end dome and therefore is electrically tied to chamber ground. A $6 \mathrm{~mm}$ vacuum gap separates the disk and outer annulus. The experiments are characterized using the cylindrical coordinate system shown in Fig. 1(d); the $\phi$ and $(R-Z)$ directions will be referred to as toroidal and poloidal, respectively. The disk and annulus each have eight $6 \mathrm{~mm}$ diameter gas injection holes spaced equally in $\phi$. The gas injection holes on the disk and annulus are at $R=4.8$ and $17.8 \mathrm{~cm}$, respectively. Details of gas injection are given in Sec. II D. The external bias magnetic field coil is located just behind the central disk but, because of the reentrant geometry, is at atmosphere. The coil consists of 110 turns of No. 11 insulated square magnetic wire wound on a phenolic form (13.0 cm inner and $18.4 \mathrm{~cm}$ outer diameters) and has an inductance of $2.3 \mathrm{mH}$ and resistance of $0.39 \Omega$. Contours of constant poloidal flux $\psi$ generated by the coil are shown in Fig. 1(d).

\section{Power systems}

The coaxial gun is powered by an ignitron-switched $120 \mu \mathrm{F}$ capacitor bank rated up to $14 \mathrm{kV}$. Eight $2 \mathrm{~m}$ long Belden YK-198 low inductance coaxial cables in parallel carry power from the capacitor bank to the gun electrodes. Routine operation at bank voltages of $5 \mathrm{kV}$ yields peak plasma currents $I_{\text {gun }} \approx 80 \mathrm{kA}$ and gun voltages $V_{\text {gun }}$ $\approx 2.5 \mathrm{kV}$ (after breakdown), or peak gun power on the order of $200 \mathrm{MW}$. Typical $I_{\text {gun }}$ and $V_{\text {gun }}$ traces are shown in Fig. 2.

The external magnetic field coil is powered by a $1.4 \mathrm{mF}$, $450 \mathrm{~V}$ capacitor bank. The half period of the current trace for the external coil is $10 \mathrm{~ms}$ and is effectively constant on the $10 \mu$ s time scale of the experiment. The bias poloidal magnetic flux $\psi_{\text {gun }}$ (intercepting the inner gun electrode) created by the external coil is typically $1-2 \mathrm{mWb}$.

\section{Gas injection}

Fast gas puffs are required in these experiments because local gas pressures on the order of $100 \mathrm{mT}$ are required for breakdown. A slow or steady gas fill is intolerable because the entire vacuum chamber would rise to $100 \mathrm{mT}$ which would result in a weakly ionized cold plasma and also an overloading of the cryopump. Fast gas valves provide a transient, highly localized cloud of high pressure gas in front of the gun electrodes. The gas valves utilize a pulsed current in a thin pancake coil to induce image currents in an adjacent aluminum disk ${ }^{20}$ but also employ a plenum for measured, reproducible gas output. The disk is thus repelled from the coil, creating a transient opening for gas flow from the plenum into the vacuum chamber. Restoring force on the disk is provided by a combination of the high pressure gas in the gas lines and a metal spring. Calibrations indicate that each pulse injects $10^{20}-10^{21}$ hydrogen molecules, depending on the applied voltage. Optimum timing of gas valve firing is determined empirically by adjusting the valve firing time to minimize the delay between capacitor bank trigger and gas breakdown (typically a few microseconds). One valve is used for the eight inner electrode gas injection holes, and two valves are used for the eight outer electrode holes. Typical gas valve firing voltage is $600 \mathrm{~V}$, with all three valves injecting on the order of $10^{21}$ hydrogen molecules. It was found that the amount of gas injected per pulse slowly increased throughout the day ${ }^{21}$ due to heating in the electrolytic capacitors $^{22}$ powering the gas valve. A new power supply using metal film polypropylene capacitors has been constructed recently to eliminate this problem. ${ }^{22}$

\section{E. Diagnostics}

The images presented in this paper were taken with multiple-frame charge-coupled device (CCD) cameras. Most of the images were taken with a Cooke Co. HSFC-PRO (Fig. 3 ). The specifications for this camera are as follows: 4 or 8 frames per shot, $1280 \times 1024$ pixels per frame, 12 bits per pixel, a $10 \mathrm{~ns}$ minimum exposure time, and a minimum interframe time of $5 \mathrm{~ns}(250 \mathrm{~ns})$ in 4 (8) shot mode. Other images were taken with a DRS Hadland Imacon 200 (Fig. 8), which has 8 or 16 frames per shot, 1200 $\times 980$ pixels per frame, 10 bits per pixel, a minimum $10 \mathrm{~ns}$ exposure time, and a minimum of $5 \mathrm{~ns}(250 \mathrm{~ns})$ interframe time in 8 (16) shot mode. The camera is placed on a tripod and positioned in front of the right-most vacuum chamber window shown in Fig. 1(c).

Magnetic field data are taken with a radial array of small pickup coils mounted on a stainless steel shaft, which can be scanned in $Z$ and rotated in $\phi$. The array contains twenty groups of three coils arranged so that all three components of B are measured from $R=0$ to $R=40 \mathrm{~cm}$ with $2 \mathrm{~cm}$ resolution. The coils are commercial chip inductors (Coil Craft $1008 \mathrm{CS}-472 \mathrm{XGBB})$ and have calibrated turns $\times$ area $N A$ $\approx 1.3 \mathrm{~cm}^{2}$. The coils are placed into precision-machined slots of a long thin strip of Delrin, which in turn is slid down a thin-wall stainless steel tube covered by an alumina tube to prevent metal contact with the plasma [see Fig. 1(a)]. The alumina has an outer diameter of $8.4 \mathrm{~mm}$. The frequency response, limited by the skin effect of the stainless steel tube, is good up to $1 \mathrm{MHz}$. Further details of probe design, construction, calibration, as well as probe improvements since this work are reported elsewhere. ${ }^{23}$

A Rogowski coil placed around the ceramic break, which is connected to the inner electrode, measures total $I_{\text {gun }}$. 
a)

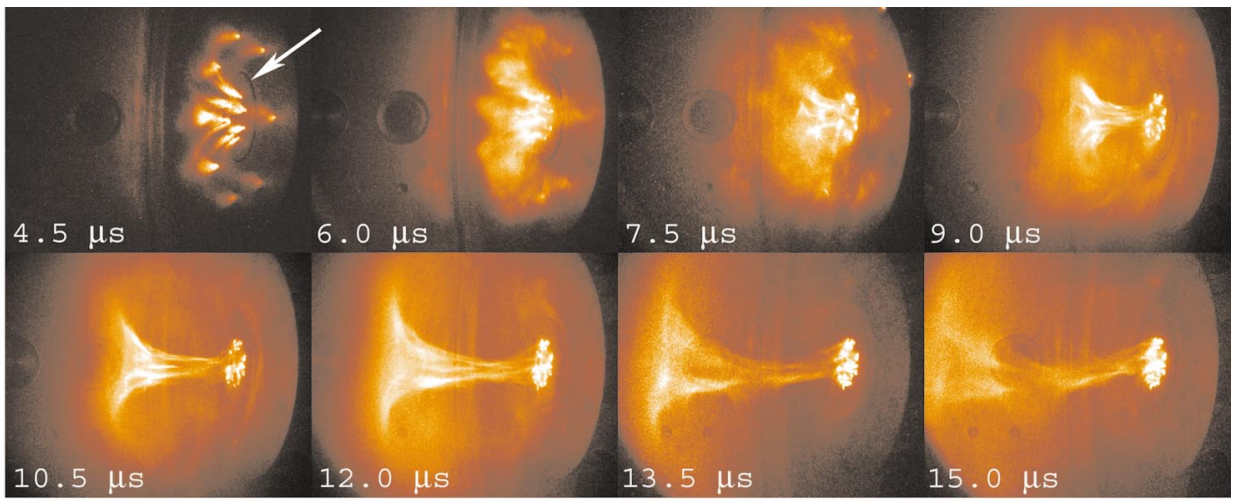

b)

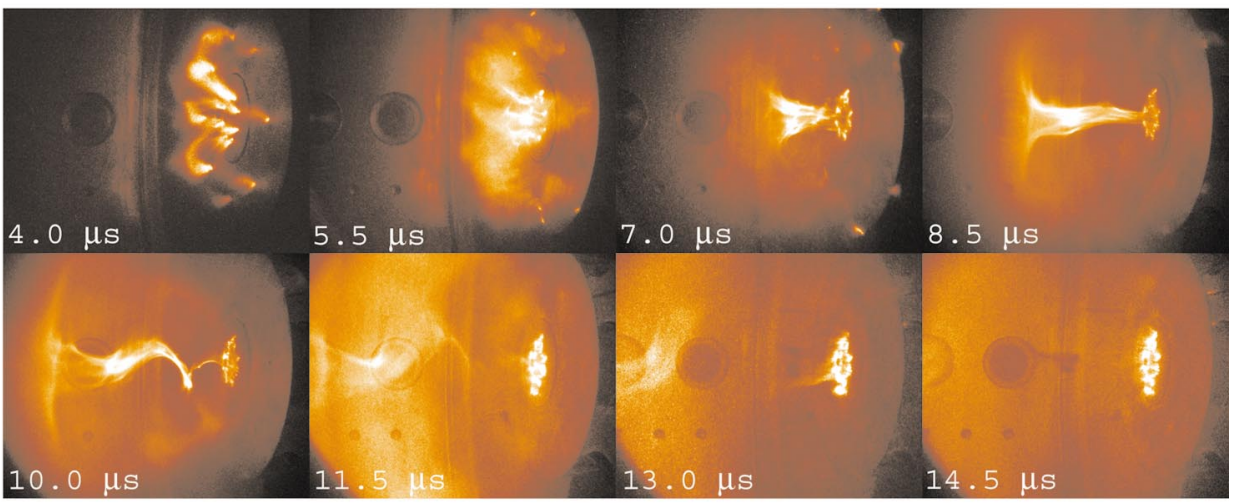

c)

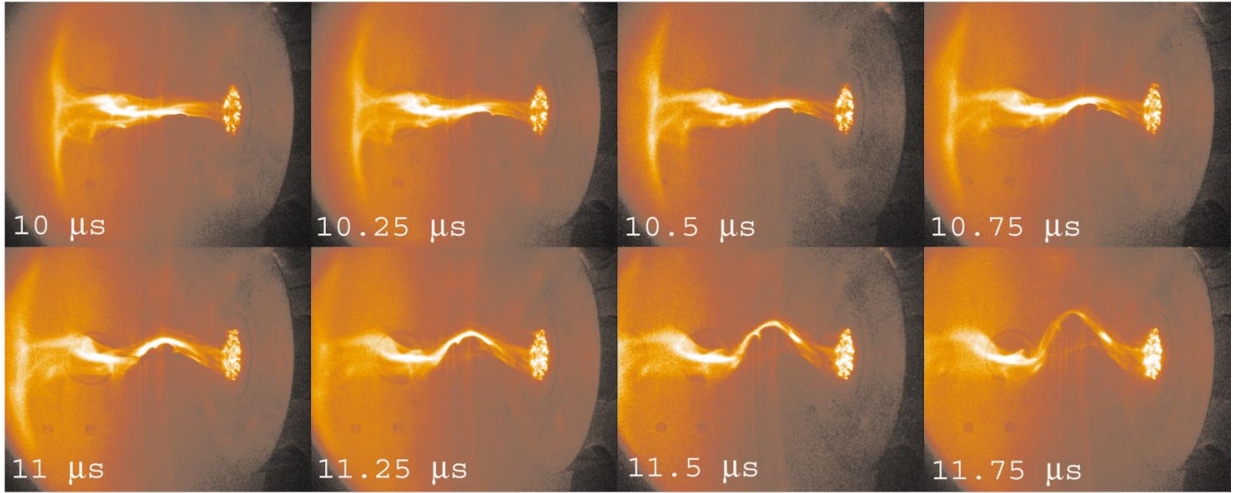

d)

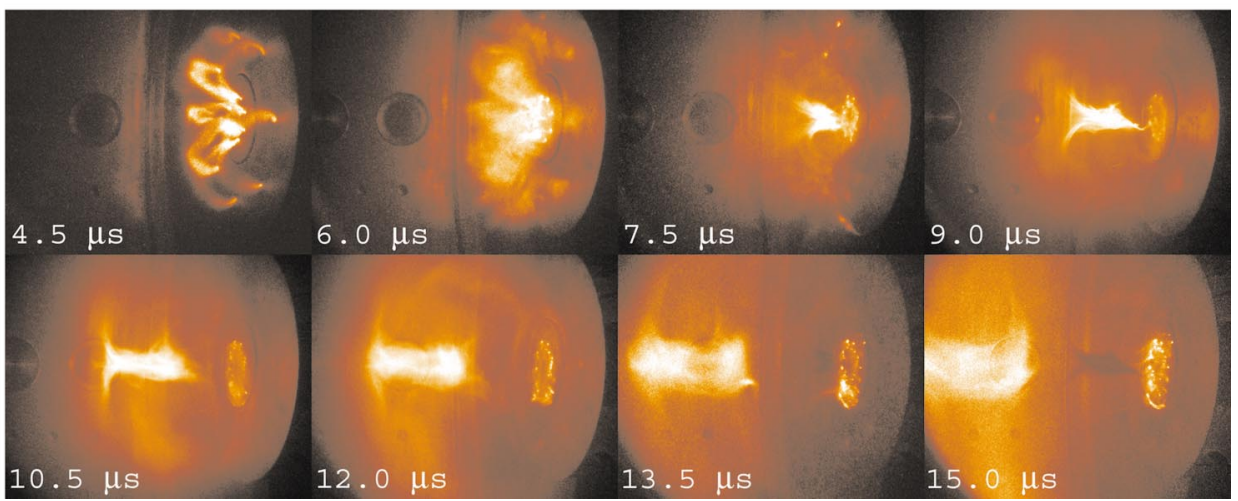

FIG. 3. (Color). CCD images of plasma evolution: (a) low $\lambda_{\text {gun }}$ regime in which a central plasma column forms (shot 1210), (b) intermediate $\lambda_{\text {gun }}$ regime in which the central column becomes helical (shot 1233), (c) kink growth (shot 1247) with $0.25 \mu$ s interframe time, and (d) high $\lambda_{\text {gun }}$ regime in which the plasma detaches from the gun before a column forms (shot 1181).

The Rogowski coil was hand-wound with a calibrated $N A$ $=9.913 \times 10^{-3} \mathrm{~m}^{2}$, and the signal is integrated via a passive integrator with $R C=8 \mathrm{~ms}$. An attenuating Tektronix P6015 high voltage probe measures $V_{\text {gun }}$.

\section{F. Control and data acquisition}

Triggering of the various power supplies (bias coil, gas valves, and coaxial gun), the data acquisition system, and the 
camera is provided by a programmable 12-channel sequencer with fiber optic outputs. A typical trigger sequence is as follows: (1) the bias coil is fired at $t=-10 \mathrm{~ms}$ (due to its $\approx 10 \mathrm{~ms}$ rise time), (2) the gas valves are fired at $t=-1.5 \mathrm{~ms}$ (to accommodate the $\approx 1.5 \mathrm{~ms}$ travel time of neutral gas through the gas lines into the plasma formation region), and (3) the main capacitor bank ignitrons are triggered at $t=0$ with gas breakdown typically occurring at $t \approx 3 \mu \mathrm{s}$.

The digital data acquisition system is a 64-channel Versa Module Europa system from Struck Innovative Systems. Each channel has a sampling rate of $105 \mathrm{MHz}$, a 12 bit resolution, $50 \Omega$ input impedance, $32 \mathrm{kB}$ memory, and an input range of $-512-512 \mathrm{mV}$ with continuously adjustable offset. All magnetic probe and gun diagnostic signals are digitized on this system. The digital acquisition system is controlled and the data is displayed and analyzed using Interactive Data Language routines. The magnetic probe array signals are integrated numerically.

\section{G. Plasma parameters}

The plasmas produced by the coaxial gun have the following nominal global parameters: $n \sim 10^{14} \mathrm{~cm}^{-3},{ }^{24} T_{\mathrm{e}} \sim T_{\mathrm{i}}$ $\sim 5-15 \mathrm{eV}$, and $B \approx 0.2-1 \mathrm{kG}$. The global plasma length scale $L$ is $\approx 25 \mathrm{~cm}$, and the typical ion gyroradius is $\rho_{i}$ $\approx 2 \mathrm{~cm}$. The characteristic Alfvén transit time is $\tau_{A}$ $\approx 1.5 \mu \mathrm{s}$; plasma lifetime is about $15 \mu \mathrm{s}$; resistive diffusion time is on the order of $1 \mathrm{~ms}$. The Lundquist number $S$ $\equiv \mu_{0} L V_{\mathrm{A}} / \eta \sim 10^{2}-10^{3}$, where $V_{\mathrm{A}}$ is the Alfvén speed and $\eta$ is the classical resistivity. Thus, the $15 \mu$ s duration plasma dynamics lasts several Alfvén times, and the magnetic flux is reasonably frozen into the plasma.

\section{EXPERIMENTAL RESULTS}

\section{A. Plasma breakdown and initial evolution}

The Paschen condition for gas breakdown ${ }^{25,26}$ shows that the voltage required for gas breakdown between two electrodes separated by distance $d$ depends on the product $p d$, where $p$ is the pressure in the region between electrodes. The dependence is such that the breakdown voltage becomes infinite when the $p d$ product becomes less than about half the value at which the breakdown voltage is minimum. This property is exploited using a fast, localized gas injection, as shown in Fig. 1(d). The gas valve operation is adjusted so that $p d$ satisfies the Paschen condition along the bias field lines. Because of the $6 \mathrm{~mm}$ size of the interelectrode gap and the negligible gas pressure in the gap, the $p d$ value in the gap is so small as to be well to the left of the Paschen minimum such that breakdown cannot occur there. This setup demonstrates the counter-intuitive fact that a very small distance between conductors in vacuum can provide perfect electrical insulation. ${ }^{27}$ This fact was also utilized in the Caltech solar prominence experimental setup. ${ }^{28}$

Breakdown along the bias field lines is shown in frame 1 of Fig. 3(a), which is a CCD camera image of the plasma within approximately the first microsecond after breakdown. The electrodes are located in the right-hand side of each frame; the circular gap between electrodes is identified by an arrow. The locations of the eight bright legs correspond to the locations of the eight pairs of gas injection holes, and the shape of the legs matches the calculated bias field configuration. These observations indicate that breakdown is occurring along the bias field lines and that the gas pressure is nonuniform and concentrated azimuthally at the eight $\phi$ positions corresponding to the gas injection holes. The breakdown occurs $\approx 2-3 \mu$ s after high-voltage is applied (see Fig. 2). In order to minimize jitter and maximize reproducibility, the gas injection timing was adjusted to minimize the time delay before breakdown.

The gun current ramps up steeply during the next $10 \mu \mathrm{s}$ (see Fig. 2), and the eight bright legs expand and merge, as shown in frames 2 and 3 of Fig. 3(a). The rising gun current corresponds to an increase in toroidal magnetic flux linking the bias field lines, and therefore magnetic helicity is injected at the rate $2 V_{\text {gun }} \psi_{\text {gun }}{ }^{26}$ The applied gun voltage creates a radial electric field. From the radial component of the ideal Ohm's law,

$$
E_{\mathrm{R}}+U_{\phi} B_{\mathrm{Z}}-U_{\mathrm{Z}} B_{\phi}=0,
$$

it is seen that a combination of toroidal rotation $U_{\phi}$ and axial flow $U_{\mathrm{Z}}$ will arise to balance $E_{\mathrm{R}}$. Note that an astrophysical accretion disk is similar to a coaxial gun but with the driving term coming from the Keplerian disk rotation $U_{\phi}$ which gives rise to $E_{\mathrm{R}} \cdot{ }^{14}$ As described below, further evolution of the plasma after frame 3 of Fig. 3(a) is dependent on the peak value of $\lambda_{\text {gun }}=\mu_{0} I_{\text {gun }} / \psi_{\text {gun }}$.

\section{B. Plasma morphologies resulting from $\lambda_{\text {gun }}$ parameter scan}

After the initial formation stage, the plasma can evolve into three distinct morphologies depending on peak $\lambda_{\text {gun. }}{ }^{14}$ Similar regimes were also observed in previous Caltech experiments using a conventional cylindrical coaxial gun. ${ }^{13}$ The parameter $\lambda_{\text {gun }}$ can be thought of as a boundary condition imposed on the plasma at the gun surface. Its functional form $\lambda_{\text {gun }}=\mu_{0} I_{\text {gun }} / \psi_{\text {gun }}$ can be easily derived by integrating $\boldsymbol{\nabla} \times \mathbf{B}=\lambda \mathbf{B}$ over the inner gun electrode surface. The parameter $\lambda_{\text {gun }}$ is varied experimentally by adjusting the main capacitor bank voltage (which controls $I_{\text {gun }}$ ) and the bias coil bank voltage (which controls $\psi_{\text {gun }}$ ). A plot of $\lambda_{\text {gun }}$ parameter space is shown in Fig. 4, with the different observed plasma morphologies (I, II, III) indicated.

\section{Formation of stable plasma column at low $\lambda_{\text {gun }}$}

The formation of a central plasma column along the $Z$ direction is observed for values of $\lambda_{\text {gun }} \lesssim 40 \mathrm{~m}^{-1}$, as shown in Fig. 3(a). Magnetic probe measurements in this regime (Fig. 5) show that the column has magnetic field radial profiles resembling a screw pinch. The axial expansion of the column is derived from Fig. 3(a) to be $\approx 40 \mathrm{~km} / \mathrm{s}$ which is of the order of the Alfvén speed. The filamentary shape of this column provides compelling experimental evidence for magnetically driven models of astrophysical jet collimation, ${ }^{12,15-17}$ in which an ionized rotating accretion disk winds up a background magnetic field and injects magnetic helicity into the disk corona. 


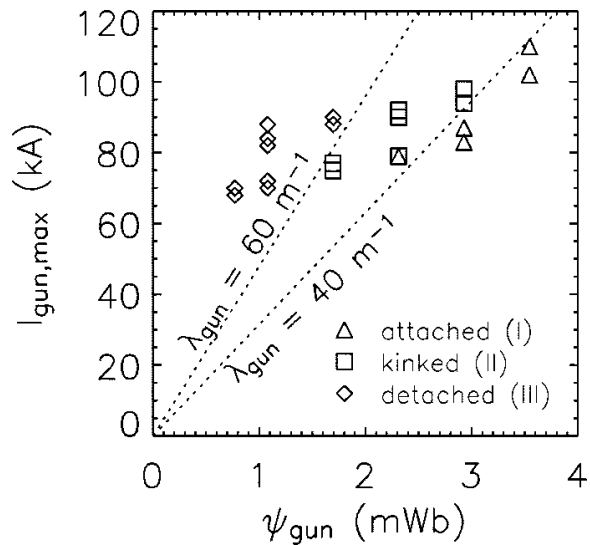

FIG. 4. Plot of $\lambda_{\text {gun }}=\mu_{0} I_{\text {gun }} / \psi_{\text {gun }}$ parameter space showing the three plasma regimes.

\section{Kink instability of central column at intermediate $\lambda_{\text {gun }}$}

For $40 \mathrm{~m}^{-1} \lesssim \lambda_{\text {gun }} \lesssim 60 \mathrm{~m}^{-1}$, a plasma column forms along the $Z$ direction but then develops a helical instability with toroidal mode number $n=1$, as shown in Figs. 3(b) and 3(c). The helical instability is shown to be consistent with an ideal MHD kink instability by two independent experimental measurements described below.

The Kruskal-Shafranov condition for MHD kink instability in cylindrical geometry is

$$
q(a)=2 \pi a B_{\mathrm{Z}}(a) / L B_{\phi}(a)<1,
$$

where $q$ is the safety factor and $a$ and $L$ are the column radius and length, respectively. By invoking the relationships $\psi_{\text {gun }} \approx \pi a^{2} B_{\mathrm{Z}}(a)$ and $B_{\phi}(a)=\mu_{0} I_{\text {gun }} / 2 \pi a=\lambda_{\text {gun }} \psi_{\text {gun }} / a$, the Kruskal-Shafranov condition for kink instability can be expressed as ${ }^{14,29}$

$$
\lambda_{\text {gun }}>4 \pi / L \text {. }
$$

Thus, the experimentally observed instability onset can be compared to the Kruskal-Shafranov condition by knowing only $\lambda_{\text {gun }}$ and $L$. Figure 6 shows a scatter plot of $\lambda_{\text {gun }}$ versus $L$ for an ensemble of shots in which a plasma column forms. The data points represent both different shots and different times within the same shot (i.e., before and after the instability occurs). It is seen that the threshold for the appearance

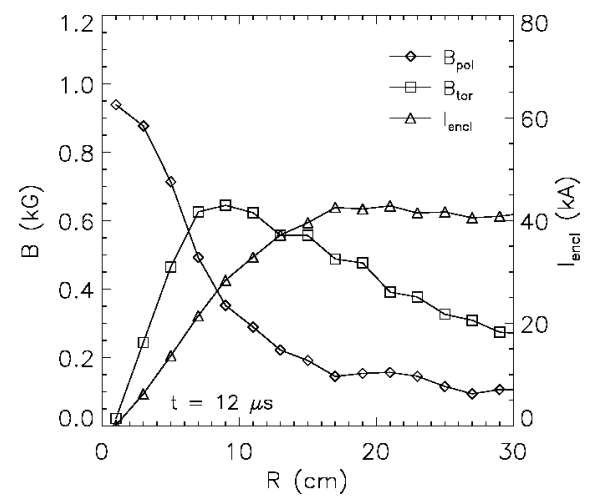

FIG. 5. Magnetic field and total current as a function of $R$ for low $\lambda_{\text {gun }}$ regime central column (shot 2465).

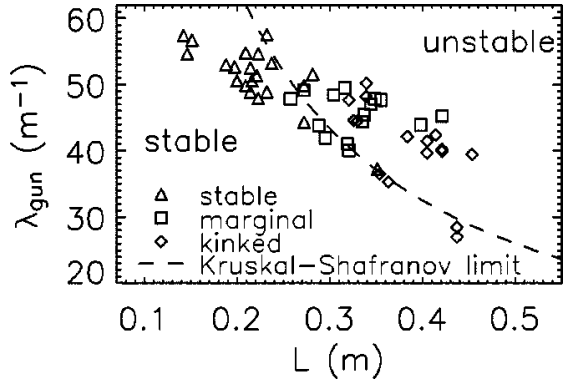

FIG. 6. Plot of $\lambda_{\text {gun }}$ vs column length $L$, showing consistency with KruskalShafranov condition $\lambda_{\text {gun }}>4 \pi / L$ for MHD kink instability.

of the helical instability is in good agreement with the Kruskal-Shafranov limit (dashed line in Fig. 6).

Consistency with the Kruskal-Shafranov limit was also checked with direct magnetic probe measurements. Radial profiles of $B_{\mathrm{Z}}$ and $B_{\phi}$ yielded $q$ profiles as a function of time, as shown in Fig. 7. It is shown that $q$ drops to unity around $10.5 \mu \mathrm{s}$. The corresponding image sequence for this shot, shown in Fig. 8, indicates that the helical instability develops at this same time. Thus the observed helical instability is consistent with an ideal kink, with onset occurring when $q$ drops to unity. The $q$ is initially greater than unity because the bias poloidal flux dominates and $L$ is small. Then $L$ increases quickly and $B_{\phi}$ also increases to a lesser degree, and therefore $q$ drops according to Eq. (2).

The growth of the kink mode was captured by shortening the interframe time of the CCD camera to $0.25 \mu \mathrm{s}$ [see Fig. 3(c)]. The mode amplitude as a function of time is shown in Fig. 9 and is seen to exhibit a fairly linear growth rate.

\section{Quick plasma detachment at high $\lambda_{\text {gun }}$}

At values of $\lambda_{\text {gun }} \geqslant 60 \mathrm{~m}^{-1}$, the plasma appears to "pinch-off" without the formation of a central column, as shown in Fig. 3(d). From the image sequence, the propagation velocity of the detached plasma along the $Z$ axis is cal-

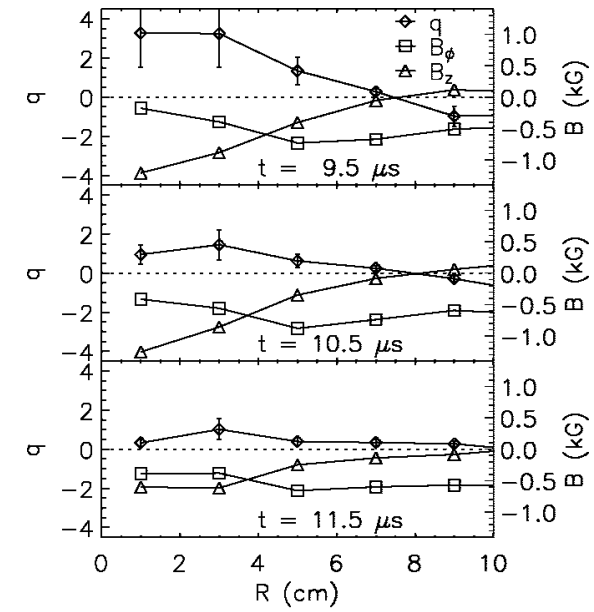

FIG. 7. Radial profiles of $q=2 \pi R B_{Z} / L B_{\phi}$ (shot 2472). The column develops a helical instability at the same time as $q$ near the axis drops to unity, also showing consistency with Kruskal-Shafranov condition for MHD kink instability. 


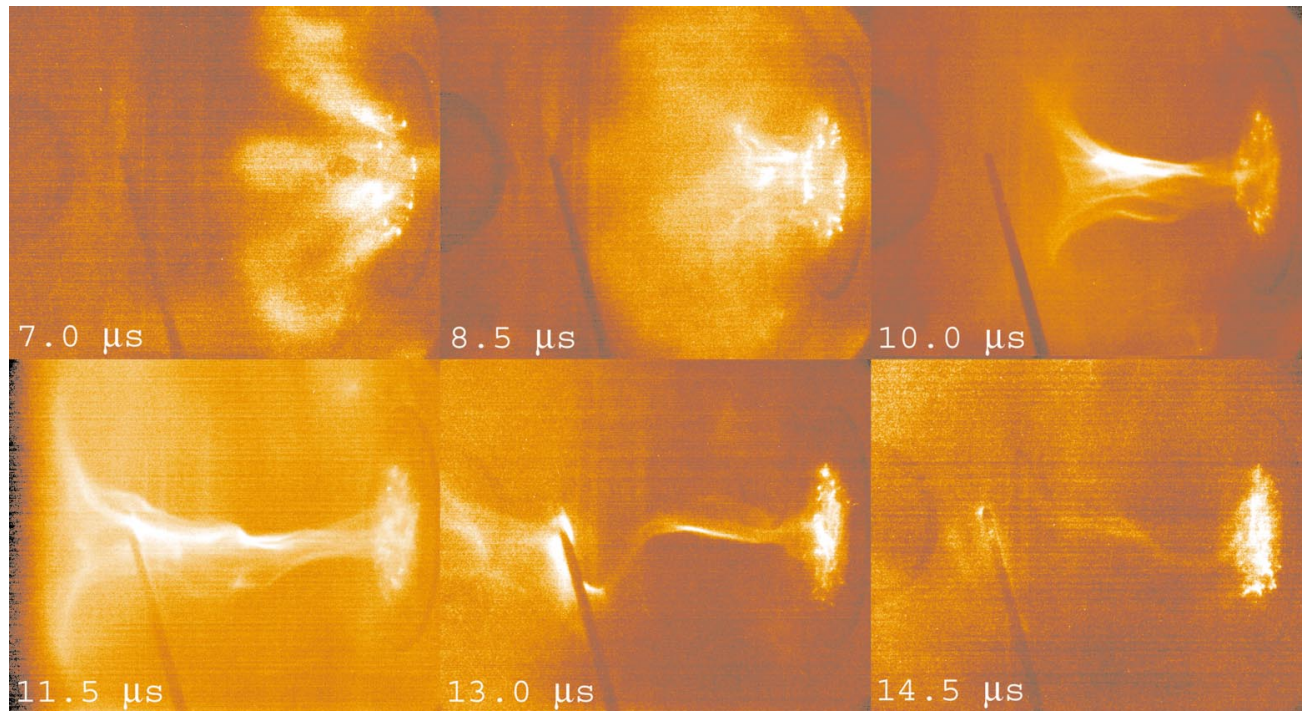

FIG. 8. (Color). CCD images of kink development for which magnetic measurements were taken (shot 2472).

culated to be around $50 \mathrm{~km} / \mathrm{s}$ (of the order of the Alfvén speed). Magnetic probe measurements indicate that the detached plasma has much stronger toroidal than poloidal field (Fig. 10). It is possible that this toroidal-flux-rich detached plasma will relax to a spheromak configuration with equivalent toroidal and poloidal flux.

Poloidal flux amplification, as measured by the magnetic probe array, is large in this regime. Flux amplification for all three regimes is shown in Fig. 11 as a function of peak $\lambda_{\text {gun }}$. In the kinked regime, the flux amplification is typically around a factor of two, and the flux amplification mechanism in this regime (discussed below) has been identified. The flux amplification is neligible in the low $\lambda_{\text {gun }}$ regime, but it is very large in the high $\lambda_{\text {gun }}$ regime. The mechanism for the high $\lambda_{\text {gun }}$ regime is not known, but it is probably related to the mechanism operating in the kink regime. An interesting question requiring further study is what determintes the maximum amount of flux amplification. The following section shows that the kink is a mechanism for poloidal flux amplification and spheromak formation.

\section{Kink as mechanism for poloidal flux amplification and spheromak formation}

The kink instability is identified experimentally as a mechanism for (1) poloidal flux amplification and (2)

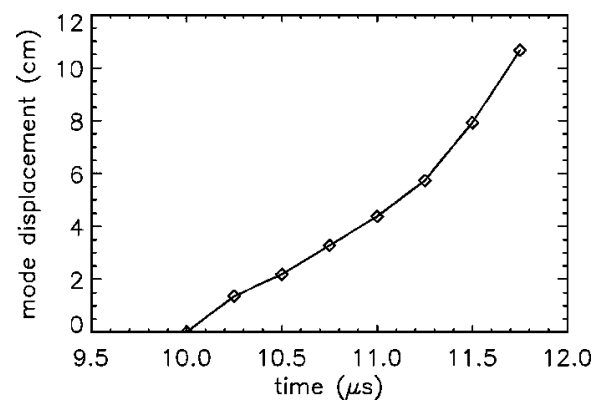

FIG. 9. Kink amplitude vs time derived from CCD image (shot 1247). spheromak formation. ${ }^{18}$ Both are experimentally observed immediately following kinking of the central column. This can be seen by examining the evolution of poloidal flux contours and maximum $\psi$ (Fig. 12) calculated from timeresolved magnetic probe data and the sequence of CCD images showing the kink (Fig. 8).

\section{Paramagnetism of the kink}

The kink modifies the direction of current flow from purely $Z$ (poloidal) to a partially $\phi$ (toroidal) direction. Consequently, it converts toroidal to poloidal flux. This process is paramagnetic, amplifying $\psi$ over the initial applied $\psi_{\text {gun }}$ $=1.7 \mathrm{mWb}$. The paramagnetism is understood by realizing that kinks involve perturbations with dependence $\exp (i \mathbf{k} \cdot \mathbf{x})$ where $\mathbf{k} \cdot \mathbf{B}=0$. The latter means

$$
k_{\mathrm{Z}}=-k_{\phi} B_{\phi} / B_{\mathrm{Z}},
$$

where $k_{\mathrm{R}}=0$. The trajectory of the kink is determined by considering a locus of constant phase of the perturbation, i.e., $\mathbf{k} \cdot \mathbf{x}=$ constant, such that

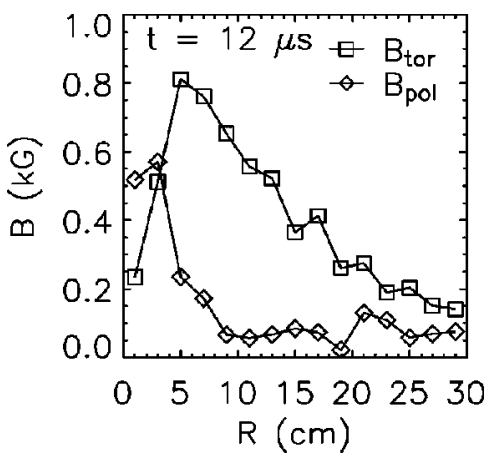

FIG. 10. Radial profiles of $B_{\text {tor }}$ and $B_{\text {pol }}$ for high $\lambda_{\text {gun }}$ plasma at $t=12 \mu \mathrm{s}$ (shot 2457). 


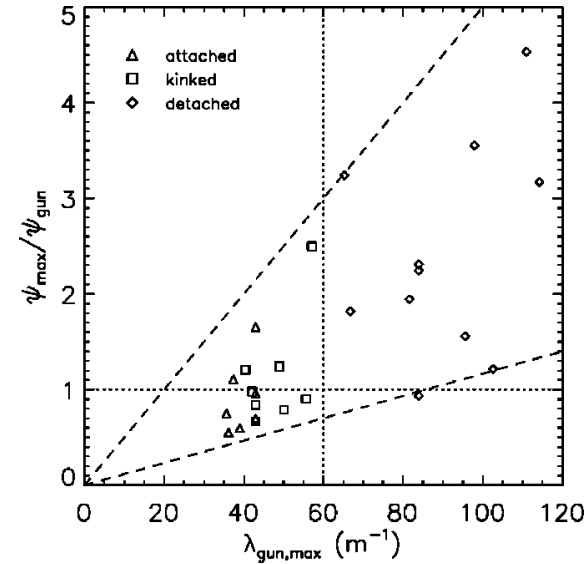

FIG. 11. Flux amplification vs peak $\lambda_{\text {gun }}$ for shots spanning all three regimes.

$$
k_{Z} Z+k_{\phi} R \phi=\text { constant } .
$$

Thus, substituting Eq. (4) into Eq. (5) gives the coordinates of the helix, $\phi=\left(B_{\phi} / R B_{\mathrm{Z}}\right) Z+$ constant, from which it can be seen that the kinked current channel is a right-handed helix if $J_{\mathrm{Z}} B_{\mathrm{Z}} \sim B_{\phi} B_{\mathrm{Z}}>0$ and a left-handed helix if $J_{\mathrm{Z}} B_{\mathrm{Z}}<0$. Thus, the helix will always have the form of a solenoid with the appropriate handedness to amplify the original $B_{\mathrm{Z}}$. The additional $\psi$ introduced by the kink can be estimated by considering the helically deformed current channel to be a solenoid with current $I$, turns per length $1 / L$, and radius $a$. The solenoid formula for the field inside the solenoid is $B_{\mathrm{Z}}=\mu_{0} I / L$; the $\psi$ produced by the solenoid is $\pi a^{2} B_{\mathrm{Z}}$ and thus depends nonlinearly on the kink amplitude $a$. Using the measured values $a \approx 5 \mathrm{~cm}, L \approx 20 \mathrm{~cm}$, and $I \approx 60 \mathrm{kA}$ at $13.5 \mu \mathrm{s}$, the $\psi$ generated by the kink is predicted to be $\approx 1 \mathrm{mWb}$, which is within a factor of 2 of the observed amplification of $\psi_{\max }$ over $\psi_{\text {gun }}$. The discrepancy is within the accuracy of $a$ and $L$ measurements and of the $\psi$ calculation assuming axisymmetry in the presence of the rotating kink. Because the coaxial gun injects only toroidal flux, 3D plasma dynamics

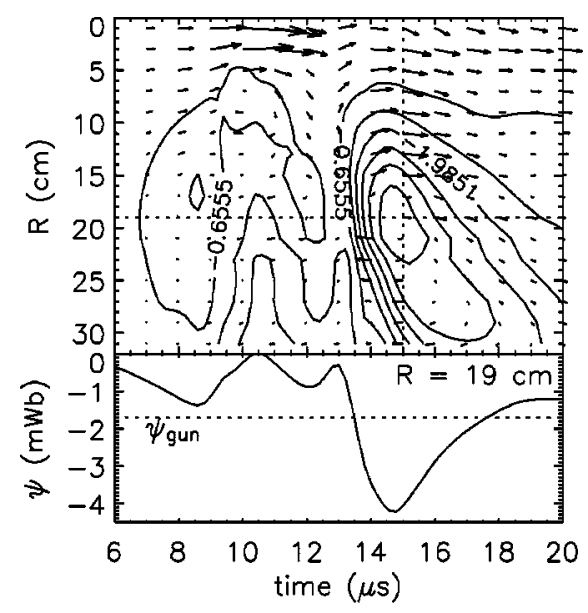

FIG. 12. Top: poloidal magnetic field vectors and $\psi$ contours as a function of $R$ and $t$. Bottom: time evolution of $\psi$ at $R=19 \mathrm{~cm}$ (shot 2472). Flux amplification is observed during time of the kink and appearance of closed $\psi$ contours.

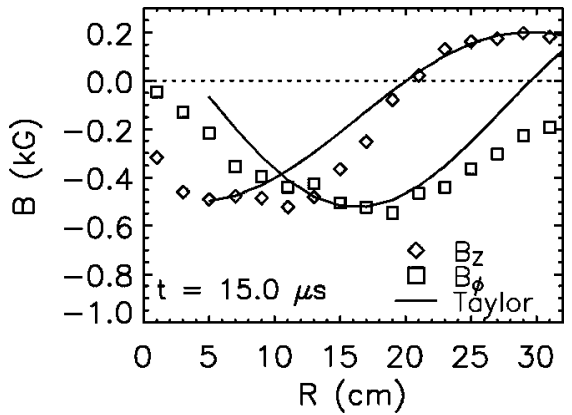

FIG. 13. Comparison of $B_{\mathrm{Z}}$ (diamonds) and $B_{\phi}$ (squares) profiles at $t$ $=15 \mu$ s with Taylor state (solid line). Profile location is indicated by vertical dashed line in top panel of Fig. 12.

must be responsible for $\psi$ exceeding $\psi_{\text {gun }}$. The dynamics are provided by the kink, which is the mechanism by which toroidal flux is converted to poloidal flux.

\section{Evidence for spheromak formation}

The appearance of the kink is followed immediately by three signatures of spheromak formation: (1) appearance of closed $\psi$-contours [calculated assuming axisymmetry, $\left.\psi(R, t)=\int_{0}^{R} 2 \pi R^{\prime} B_{\mathrm{Z}}\left(R^{\prime}, t\right) \mathrm{d} R^{\prime}\right],(2) \psi$-amplification, and (3) magnetic field radial profiles consistent with spheromak formation. It is important to note that the relationship between closed $\psi$ contours and closed flux surfaces becomes ambiguous when axisymmetry is broken. Thus, in the presence of a nonaxisymmetric kink, closed $\psi$ contours indicate closed flux surfaces only in a time-averaged manner. Shortly after $13 \mu \mathrm{s}$, the kink breaks apart (Fig. 8). This coincides with signatures of spheromak formation as observed in the magnetic probe measurements (Fig. 12). At $\approx 13 \mu$ s, closed $\psi$ contours appear and $\psi_{\max }$ is amplified to larger than $\psi_{\text {gun }}(\psi$ amplification is due mainly to broadening of the $B_{\mathrm{Z}}$ profile after $12 \mu \mathrm{s})$. At $15 \mu \mathrm{s}$, magnetic field profiles consistent with spheromak formation are observed (Fig. 13). The measured radial profiles of $B_{Z}$ and $B_{\phi}$ at $15 \mu$ s are compared with Taylor state solutions ${ }^{7}$ in cylindrical geometry, i.e., uniform $\lambda$ solutions of $\boldsymbol{\nabla} \times \mathbf{B}=\lambda \mathbf{B}$. The solutions are $B_{\mathrm{Z}}$ $\sim J_{0}(\lambda R)$ and $B_{\phi} \sim J_{1}(\lambda R)$, where $J_{0}$ and $J_{1}$ are Bessel functions of order 0 and 1 , respectively, and the best fit is found for $\lambda \approx 15 \mathrm{~m}^{-1}$ with a radial offset of $4 \mathrm{~cm}$ (displacement of spheromak off the geometric axis). The slight disagreement between measured profiles and the Taylor solution is not surprising since the spheromak is expected to be either (1) still undergoing relaxation toward the Taylor state or (2) in a modified relaxed state since it is still being driven by the gun, where peak $\lambda_{\text {gun }} \approx 50 \mathrm{~m}^{-1}$.

\section{DISCUSSION}

\section{A. Spheromak formation and topology}

Spheromak formation involves a sequence of steps that in principle should be possible to visualize mentally. However, the inherent geometric and topological complexity of these steps has made such a visualization so challenging that no consensus exists on the precise form of this sequence. This shortcoming is important because without being able to 
visualize how the 3D topology evolves during the spheromak formation sequence, it is difficult to optimize this process and the closely related sustainment process. ${ }^{30}$ Because visualization of the process has proved difficult, the main appoach used to date for experimentally characterizing spheromak formation and sustainment is modal analysis, a method where Fourier modes are first identified and then the time dependence and spatial profile of these modes are measured and compared with theoretical models. While useful in many respects, modal analysis gives little insight into 3D topological issues such as linkage, connectivity, knottedness, and conversion between toroidal flux and poloidal flux. The photographs of evolving magnetized plasmas in the present experiments have provided an actual visualization which helps to provide insights into these issues. Interpretations given here are placed into context with respect to past experimental work. ${ }^{9,29,31-34}$ It should also be noted that many aspects of the present work are qualitatively similar to recent timedependent MHD simulations of spheromak formation via electrostatic helicity injection. ${ }^{35}$

Bounded, axisymmetric, force-free plasma equilibria were first discussed in the 1950s. ${ }^{3,36-38}$ These theoretical models invoked rather abstract astrophysical contexts and did not contain descriptions of the detailed processes by which such equilibria could be created. A hypothetical analogy to this situation would be to be in possesion of an abstract theoretical model demonstrating that soap bubbles should in principle exist, while never having observed a soap bubble nor being aware of how to blow soap bubbles. The soap bubble analogy is apt because a bounded, axisymmetric, force-free equilibrium can be thought of as being a bubble (local maximum) of poloidal flux $\psi(R, Z)$. First derivatives (gradients) of $\psi$ show that a local $\psi(R, Z)$ maximum constitutes a magnetic axis, and second derivatives (Laplacian-like operators) of $\psi$ show that a toroidal current necessarily flows along this magnetic axis.

Lacking detailed knowledge for how to make these "magnetic bubbles" is a nontrivial shortcoming because Cowling's antidynamo theorem ${ }^{39}$ shows that there is no simple way to make such $\psi$ bubbles. Specifically, Cowling showed that creation/sustainment of an axisymmetric, steady-state toroidal current cannot be achieved via axisymmetric (i.e., simple) means. Since creation of an axisymmetric toroidal current is tantamount to creating a local $\psi(R, Z)$ maximum, the Cowling antidynamo theorem effectively requires that any poloidal flux amplification process must be nonaxisymmetric. Thus, spheromak formation and sustainment necessarily involve nonaxisymmetric processes.

Helical (i.e., nonaxisymmetric) distortions of magnetized coaxial gun central columns and associated poloidal flux amplification were first observed in the early 1960s in experiments by Alfvén et al. ${ }^{8}$ and Lindberg and Jacobsen. ${ }^{9}$ It is interesting to note that these early magnetized coaxial gun experiments revealed phenomena relating to spheromak formation nearly two decades before the word "spheromak" was coined by Rosenbluth and Bussac. ${ }^{40}$ In particular, Lindberg and Jacobsen ${ }^{9}$ imaged the helical instability of an initially azimuthally symmetric axial current channel, characterized this as an " $m=1$ instability," noted that "such instabilities are well known in the theories of pinches," and measured an amplification of the poloidal flux. Lindberg et al. interpreted this central column helix as a kink instability of the geometric axis current and postulated that the helical deformation of the current path was responsible for observed poloidal flux amplification. However, Lindberg et al. did not provide quantitative measurements establishing that the observed helical instability was indeed a kink (i.e., that the configuration destabilized when the $q=1$ kink stability threshold was crossed) nor that the instability produced a magnitude and polarity of poloidal flux consistent with observation.

Similar modes (now called $n=1$ modes) were also observed in all later coaxial gun experiments, ${ }^{31-33,41}$ but there have been two distinct, differing interpretations of these modes. In the first interpretation, referred to here as "rotating static mode," the modes are assumed to have a steady-state geometry in some rest frame so that their apparent laboratory frame time-dependence is solely due to the rotation of the mode rest frame with respect to the laboratory frame. In this interpretation the modes are thus assumed to have a laboratory frame time dependence $\sim \cos (n \theta-\omega t)$ so that an observer located in a frame rotating with the mode (i.e., a frame with coordinate $\left.\theta^{\prime}=\theta-\omega t / n\right)$ would see a time independent, or static, mode. In the second interpretation, referred to here as "relaxation oscillation," the modes are not static in any frame but, rather, are growing modes which start with zero amplitude, build up to finite amplitude, undergo a nonlinear crash, and then the process repeats. There might be rotation as well, but even if one moved to an optimally chosen rotating frame, there still would be relaxation oscillations. Thus, in the relaxation oscillation case, the mode time-dependence during its growth stage would be $\sim e^{\gamma t} \cos n \theta$ or possibly $\sim e^{\gamma t} \cos (n \theta-\omega t)$ so that no change of observer rest frame would cause the mode to appear static.

The $n=1$ modes were typically observed as oscillations in magnetic field during the sustainment (as opposed to formation) phase. However, it seems likely that sustainment ought to involve much the same processes as formation since formation requires creating a localized poloidal flux maximum, and sustainment requires maintaining this maximum against resistive losses. Knox et al. ${ }^{31}$ observed $n=1$ and higher order modes using wall magnetic probe arrays and proposed that sustainment was due to a rotating static mode which they called a "rotating internal kink distortion." On the other hand Nagata et al. ${ }^{33}$ made similar magnetic probe measurements but interpreted sustainment as a sequence of relaxation oscillations (collapse and recovery). Thus Knox et $a l$. considered the mode as a rotating perturbation of an otherwise axisymmetric flux surface, whereas Nagata et al. interpreted the mode as being the creation and destruction of flux surfaces.

The SPHEX group ${ }^{29,32,34}$ used internal probes to identify two distinct structures in their experiment, namely, (i) a central column of open magnetic field lines with a force-free current flowing from the gun electrode along the geometric axis and (ii) an annulus (torus) of closed field lines and toroidal current linking the central column. The SPHEX group found that the $n=1$ oscillations were primarily in the central column and, using probes, showed that the central column 
had a helical deformation. The helical deformation was inferred on the assumption that the $n=1$ oscillation was a rotating static mode, and this mode was interpreted to be a nonlinearly saturated kink instability of the geometric axis current rotating toroidally at the $E \times B$ frequency. ${ }^{42}$ It is important to emphasize that the rotation was assumed ${ }^{29}$ but not directly measured. Because the signal was observed to be periodic, it was assumed that the fluctuations were of the form $\cos (\theta-\omega t)$ so that observations made at different times could be used to map out the spatial profile of the assumed rotating structure. However, this assumption of rotation misses the interpretation that there is a relaxation oscillation with repeated births of new helices, i.e., the rotation assumption did not consider the possibility of a repetitive sequence of modes with time behavior $e^{\gamma t} \cos \theta$.

The present results suggest that the $n=1$ fluctuations result from repeated births of new kinked helices with time dependence $\sim e^{\gamma t} \cos \theta$ and that these helices detach and merge into the time-averaged closed flux region of a spheromak. According to this interpretation of spheromak formation/sustainment, the current starts out aligned along the geometric axis and so is initially purely poloidal. Then, upon kinking (i.e., the current channel becoming helical so that it is effectively a solenoid), the current channel axis has a toroidal component which creates new poloidal flux. Kink growth means that the radius of the helix increases. Growth of the kink, in turn, means that the radius of the helix increases until this radius becomes so large that the helical current channel merges with any existing spheromak toroidal current, thereby amplifying/sustaining the poloidal flux. New current will now flow from the gun along a new straight central column aligned along the geometric axis, and initially, this new central current column will be stable against kinking because of the strong axial magnetic field resulting from the strong toroidal current flowing in the spheromak. However, because no electromotive force sustains the spheromak toroidal current against Ohmic decay, the spheromak toroidal current decays with time so that the poloidal field on the geometric axis becomes weaker. The open poloidal field on the geometric axis weakens to the point that a kink develops on the geometric axis. The new kink deforms into a new solenoid which merges with the spheromak, replenishing the toroidal curent in the spheromak, and increasing the geometric axis open poloidal field so that the process starts over again. Thus, power flow from the geometric axis region to the spheromak is not the result of rotation of a saturated kink but is instead due to the repetitive creation of successive helical (kinked) geometric axis current channels which expand nonlinearly in radius and merge with the spheromak.

The interpretation in the above paragraph is buttressed by considering what happens when a simple solenoid, consisting of many turns of wire, is rotated about its own axis. Such a rotation does not result in flow of magnetic energy or in transformation of toroidal field energy into poloidal field energy. In fact rotation of a solenoid about its axis does not change the magnetic field produced by the solenoid at all because rotation of a solenoid does not change the relative toroidal drift velocity between electrons and ions in the so- lenoid wire. In contrast, increasing the radius of the solenoid, as would be the consequence of a growing kink instability, increases the poloidal flux through the bore of the solenoid. If the conductor forming the solenoid (i.e., the initial geometric axis current channel) is a flux conserver, then this increase of poloidal flux linking the conductor must be at the expense of a decrease in the toroidal flux linking the conductor, resulting in a conversion from toroidal to poloidal flux due to the kink.

The primary result in this work regarding spheromak formation is the experimental demonstration that the kink instability of the geometric axis current and its growth result in the poloidal flux amplification required to produce the local maximum of poloidal flux necessary for spheromak formation. This result is qualitatively similar to the point of view expressed by Lindberg et al. and Nagata et al. but differs significantly from the point of view expressed by Knox et al. and the SPHEX group. The present work has provided a quantitative demonstration that the onset of the helical instability is consistent with the Kruskal-Shafranov condition for kink instability and a theoretical demonstration that the kink is necessarily paramagnetic (i.e., amplifies poloidal flux). Furthermore, it was shown that the poloidal flux amplification calculated using the visually observed kink dimensions are consistent with the magnetic probe measurements of poloidal flux. These measurements support the interpretation that poloidal flux amplification is due to kink growth and not kink rotation. Repetition of this sequence with successive, new kinked current channels during sustainment could explain the previously observed $n=1$ fluctuations. The flux amplification mechanism would be insensitive to the magnitude or direction of the rotation frequency.

\section{B. Astrophysical jets}

Magnetically driven astrophysical jets are intriguing astronomical phenomena which have been observed for nearly a century. These jets are highly collimated and have been observed to emanate from stars, black holes, and active galactic nuclei; the sources are generically called central objects. The characteristic size of jets ranges over many orders of magnitude, but in all cases the axial extent of the jet is much larger than the characteristic dimension of the source. Theoretical models of jet formation and collimation have been discussed at length in the literature. ${ }^{10-12}$ Observations of helical magnetic fields in jets ${ }^{43}$ provide especially tantalizing evidence that plasma physics plays an important role governing jet collimation, structure, and dynamics. Theoretical models based on MHD have become the leading candidates to explain jet formation and collimation. Many of these models show how axisymmetric force-free or nearly forcefree plasma equilibria could be made to resemble the long thin morphology of a jet. ${ }^{15,44,45}$ More recently, timedependent 3D MHD simulations ${ }^{12,16}$ have illustrated dynamical collimation starting with an initial "seed" magnetic field configuration. Despite impressive similarities between the theoretical models and astronomical observations of jets, experiments to test the underpinnings of these theories have been lacking. 
The planar spheromak gun formation experiment discussed here has a geometry very similar to the geometry associated with the formation region of magnetically driven astrophysical jets [Fig. 1(b)], and so this work can be considered as a laboratory investigation into the underlying processes governing astrophysical jet formation, collimation, and acceleration. ${ }^{14}$ The MHD theories are based on the generation of an axisymmetric poloidal current with geometry essentially the same as the poloidal current in the central column of the present experiments. In the astrophysical situation, this poloidal current is driven by a radial electric field resulting from the rotation of an accretion disk located in the equatorial plane of the central object. The rotation of the accretion disk cuts across a pre-existing poloidal magnetic field, resulting in an electric field $\mathbf{E}=-U_{\phi} \hat{\phi} \times B_{Z} \hat{Z}$ $=-U_{\phi} B_{Z} \hat{R}$, which is equivalent to the radial electric field applied across the gap between inner and outer electrodes in the experiment. In both cases, magnetic helicity is injected at the rate $\mathrm{d} K / \mathrm{d} t=2 V \psi$, where $V$ is the potential drop between the inner and outer electrodes (or between star and disk) and $\psi$ is the poloidal magnetic flux linking the two.

The present work demonstrates experimentally that magnetic helicity injection in relevant geometry leads to the formation and collimation of a jet [Fig. 3(a)]. In particular, the data indicate both a jetlike flow and a collimation of this flow, i.e., the lengthening central column is the jet, and the narrowing of the central column is the collimation. The jet flow is consistent with a recent model ${ }^{17}$ proposing that axial gradients in $B_{\phi}^{2}$ drive axial flows from where the current channel radius is small to where the channel radius is large and that collimation occurs where $\boldsymbol{\nabla} \cdot \mathbf{U}$ is negative so that there is a compression of the toroidal flux frozen into the flow. The present work also shows that collimation can occur within just one twist of the poloidal magnetic field, as demonstrated by the fact that the kink instability occurs when $q$ drops to unity, after collimation has already been observed. It is interesting to note that the kink instability has been proposed to explain the observed knotted structures in astrophysical jets. ${ }^{46}$

\section{SUMMARY}

Magnetic helicity injection experiments using a simple magnetized coaxial gun setup have revealed insights into the dynamics of spheromak formation and collimation of magnetically driven astrophysical jets. Depending on the peak value of $\lambda_{\text {gun }}$, three different plasma morphologies were observed: (1) a collimated jet at $\lambda_{\text {gun }} \lesssim 40 \mathrm{~m}^{-1}$, (2) kink instability of the jet at $40 \leq \lambda_{\text {gun }} \leq 60 \mathrm{~m}^{-1}$, and (3) a quickly detached plasma at $\lambda_{\text {gun }} \geqslant 60 \mathrm{~m}^{-1}$. The kink instability was shown to provide the mechanism for poloidal flux amplification in coaxial gun spheromak formation. The latter result supports a model in which repeated births of kinked helices can form and sustain spheromaks. It is proposed that this mechanism, rather than a rotating static saturated kink which does not lead to flux amplification, could explain past observations of $n=1$ fluctuations in coaxial gun spheromak experiments. A collimated jet is formed by driving electric current along a background poloidal field in a geometry relevant for magnetically driven astrophysical jets. Collimation is observed well before kink onset. Since kink onset takes place when the magnetic field has undergone one complete twist, consistent with the Kruskal-Shafranov theory, these observations demonstrate that collimation occurs before the initial poloidal field has undergone one complete twist. Kink dynamics could be related to wiggled structures in astronomical observations of galactic jets.

\section{ACKNOWLEDGMENTS}

The authors acknowledge S. Pracko, C. RomeroTalamás, F. Cosso, and D. Felt for technical assistance and also Dr. S. Woodruff for discussions about past work on spheromak $n=1$ central column instabilities.

This work was supported by U.S. Department of Energy (DOE) Fusion Energy Sciences Postdoctoral Fellowship and DOE Grant No. DE-FG03-98ER544561.

${ }^{1}$ S. Lundquist, Ark. Fys. 2, 361 (1950).

${ }^{2}$ S. Chandrasekhar and L. Woltjer, Proc. Natl. Acad. Sci. U.S.A. 44, 285 (1958).

${ }^{3}$ L. Woltjer, Proc. Natl. Acad. Sci. U.S.A. 44, 489 (1958).

${ }^{4}$ M. A. Berger and G. B. Field, J. Fluid Mech. 147, 133 (1984).

${ }^{5}$ J. B. Taylor, Phys. Rev. Lett. 33, 1139 (1974).

${ }^{6}$ W. C. Turner, G. C. Goldenbaum, E. H. A. Granneman, J. H. Hammer, C. W. Hartman, D. S. Prono, and J. Taska, Phys. Fluids 26, 1965 (1983).

${ }^{7}$ J. B. Taylor, Rev. Mod. Phys. 58, 741 (1986).

${ }^{8}$ H. Alfvén, L. Lindberg, and P. Mitlid, J. Nucl. Energy, Part C 1, 116 (1960).

${ }^{9}$ L. Lindberg and C. Jacobsen, Astrophys. J. 133, 1043 (1961).

${ }^{10}$ M. C. Begelman, R. D. Blandford, and M. J. Rees, Rev. Mod. Phys. 56, 255 (1984)

${ }^{11}$ R. D. Blandford, Philos. Trans. R. Soc. London, Ser. A 358, 811 (2000).

${ }^{12}$ D. L. Meier, S. Koide, and Y. Uchida, Science 291, 84 (2001).

${ }^{13}$ J. Yee and P. M. Bellan, Phys. Plasmas 7, 3625 (2000).

${ }^{14}$ S. C. Hsu and P. M. Bellan, Mon. Not. R. Astron. Soc. 334, 257 (2002).

${ }^{15}$ D. Lynden-Bell, Mon. Not. R. Astron. Soc. 279, 389 (1996).

${ }^{16}$ R. Ouyed, D. A. Clarke, and R. E. Pudritz, Astrophys. J. 582, 292 (2003).

${ }^{17}$ P. M. Bellan, Phys. Plasmas 10, 1999 (2003).

${ }^{18}$ S. C. Hsu and P. M. Bellan, Phys. Rev. Lett. 90, 215002 (2003).

${ }^{19}$ S. C. Hsu and P. M. Bellan, IEEE Trans. Plasma Sci. 30, 10 (2002).

${ }^{20}$ J. C. Thomas, D. Q. Hwang, R. D. Horton, J. H. Rogers, and R. Raman, Rev. Sci. Instrum. 64, 1410 (1993).

${ }^{21}$ J. Yee, Ph.D. thesis, Caltech, 1999.

${ }^{22}$ P. M. Bellan, Rev. Sci. Instrum. 73, 2900 (2002).

${ }^{23}$ C. A. Romero-Talamás, P. M. Bellan, and S. C. Hsu, Rev. Sci. Instrum. 75, 2664 (2004).

${ }^{24}$ Much higher local density observed on axis. G. Yun (private communication).

${ }^{25}$ A. Von Engel, Ionized Gases (Oxford University Press, Oxford, 1965).

${ }^{26}$ P. M. Bellan, Spheromaks (Imperial College Press, London, 2000).

${ }^{27}$ C. Braun, W. Hartmann, V. Dominic, G. Kirkman, M. Gundersen, and G. McDuff, IEEE Trans. Electron Devices 35, 559 (1988).

${ }^{28}$ P. M. Bellan and J. F. Hansen, Phys. Plasmas 5, 1991 (1998).

${ }^{29}$ R. C. Duck, P. K. Browning, G. Cunningham, S. J. Gee, A. al Karkhy, R. Martin, and M. G. Rusbridge, Plasma Phys. Controlled Fusion 39, 715 (1997).

${ }^{30}$ S. Woodruff, D. N. Hill, B. W. Stallard, R. Bulmer, C. T. Holcomb, E. B. Hooper, H. S. McLean, J. Moller, and R. D. Wood, Phys. Rev. Lett. 90, 095001 (2003).

${ }^{31}$ S. O. Knox, C. W. Barnes, G. J. Marklin, T. R. Jarboe, I. Henins, H. W. Hoida, and B. L. Wright, Phys. Rev. Lett. 55, 842 (1986).

${ }^{32}$ P. K. Browning, G. Cunningham, S. J. Gee, K. J. Gibson, A. al Karkhy, D. A. Kitson, R. Martin, and M. G. Rusbridge, Phys. Rev. Lett. 68, 1718 (1992).

${ }^{33}$ M. Nagata, T. Kanki, T. Masuda, S. Naito, H. Tatsumi, and T. Uyama, Phys. Rev. Lett. 71, 4342 (1993).

${ }^{34}$ D. M. Willet, P. K. Browning, S. Woodruff, and K. J. Gibson, Plasma Phys. Controlled Fusion 41, 595 (1999). 
${ }^{35}$ C. R. Sovinec, J. M. Finn, and D. del Castillo-Negrete, Phys. Plasmas 8, 475 (2001).

${ }^{36}$ L. Woltjer, Proc. Natl. Acad. Sci. U.S.A. 44, 833 (1958).

${ }^{37}$ S. Chandrasekhar, Proc. Natl. Acad. Sci. U.S.A. 42, 1 (1956).

${ }^{38}$ S. Chandrasekhar and P. C. Kendall, Astrophys. J. 126, 457 (1957).

${ }^{39}$ T. G. Cowling, Mon. Not. R. Astron. Soc. 94, 39 (1934).

${ }^{40}$ M. N. Rosenbluth and M. N. Bussac, Nucl. Fusion 19, 489 (1979).

${ }^{41}$ T. R. Jarboe, Plasma Phys. Controlled Fusion 36, 945 (1994).

${ }^{42}$ D. Brennan, P. K. Browning, R. A. M. V. der Linden, A. W. Hood, and S.
Woodruff, Phys. Plasmas 6, 4248 (1999).

${ }^{43}$ K. Asada, M. Inoue, Y. Uchida, S. Kameno, K. Fujisawa, S. Iguchi, and M. Mutoh, Publ. Astron. Soc. Jpn. 54, L39 (2002).

${ }^{44}$ H. Li, R. V. E. Lovelace, J. M. Finn, and S. A. Colgate, Astrophys. J. 561, 915 (2001).

${ }^{45}$ R. V. E. Lovelace, H. Li, A. V. Koldaoba, G. V. Ustyugova, and M. M. Romanova, Astrophys. J. 572, 445 (2002).

${ }^{46}$ M. Nakamura, Y. Uchida, and S. Hirose, New Astron. 6, 61 (2001). 